\title{
5 Spanisch lernen in Berlin: von der Kita bis zum Abitur
}

„Warum lernen wir fremde Sprachen?“ Unter diesem Titel veröffentliche 1934 der vielseitig interessierte Philologe, Jurist und Schriftsteller Philipp Krämer ${ }^{18}$ ein Manuskript von einem „Vortrag gehalten auf der Tagung der Lehrer für neuere Sprachen“ (so der Untertitel), die zuvor in Finnland stattgefunden hatte. Die Titelfrage stellt sich auch heute in derselben Form immer wieder. Krämer beantwortet sie auf neun Seiten in einer Weise, die erstaunlich aktuell geblieben ist. Sein Hauptargument: Sprachen sollten nicht aus einer utilitaristischen oder gar materialistischen Motivation heraus gelernt werden, sondern aus kulturellen und historischen Gründen. Sprachenlernen mache die historischen Verbindungen und die kulturellen Unterschiede und Gemeinsamkeiten mit Anderen zugänglich und verstehbar, und biete so auch einen besseren Zugang zum Verständnis der vielfältigen Facetten, die die eigene Kultur ausmachten. Entgegen der politischen Stimmung der Zeit bedeutet dies für ihn in Deutschland: „Wenn der deutsche Geist in seiner Geschichte mit Hellas und Rom, mit Frankreich und England auf die geschilderte Weise schöpferisch zusammengeschlossen ist, dann kann es für die deutsche höhere Schule in erster Linie nur folgende fremde Sprachen geben: Latein, Griechisch, Französisch, Englisch“ (Krämer 1934: 255). Abgesehen vom Griechischen, dessen Position inzwischen stark geschwächt ist, bleiben die genannten kanonischen Fremdsprachen bis heute bedeutsam. Krämer fährt fort mit einer Beobachtung, in der zwei weitere Sprachen genannt werden, die ebenfalls heute im Fremdsprachenangebot Beachtung erfahren: „Der Kaufmann meldete schon immer Spanisch und Russisch an“ (Krämer 1934: 255). Die Verknüpfung der beiden Sprachen mit ökonomischen Interessen mag heute überraschen, gilt doch Englisch als die globale Wirtschaftssprache und als Karrierekriterium par excellence.

Dennoch illustriert die Aufstellung möglicher Fremdsprachen hier eine interessante Überlegung, die schon vor fast einhundert Jahren diskutiert wurde und die bis heute zentral bleibt: Welchen Platz sollte man welchen Sprachen in der Schule zuweisen? Mit Blick auf das Spanische wird deutlich, dass es - ähnlich wie auch das Russische nach der Wiedervereinigung - anscheinend einer besonderen Rechtfertigung bedarf, weil es nicht zum engeren Kreis der kanonischen Fremdsprachen im deutschen Schulsystem gerechnet wird. Zugleich sind inzwischen aber auch die klassischen Sprachen und sogar das Französische immer

18 Die Namensgleichheit mit dem Autor des Buches ist rein zufällig.

Ә Open Access. ( 2020 Philipp Krämer, publiziert von De Gruyter. (cc)BY Dieses Werk ist lizenziert unter der Creative Commons Attribution 4.0 International Lizenz. 
weniger selbstverständlich, und nur das Englische wird allgemein als notwendig anerkannt. Wie das Spanische in diesem sich wandelnden Gefüge verortet wird, lohnt deshalb einen genaueren Blick. Dabei bietet es sich an, dem Bildungsweg im Berliner Schulsystem vom vorschulischen Bereich bis zur Hochschulreife zu folgen. Die späteren Bildungsbereiche werden in den darauffolgenden Teilen im Rahmen der Befragungsstudien behandelt.

\subsection{Kindergarten}

Der Bereich der vorschulischen Bildung ist in Berlin in ständiger Bewegung, insbesondere was mehrsprachige Angebote betrifft. Immer mehr Kitas werden auf Initiative von Eltern gegründet, die bestimmte Erziehungskonzepte oder Schwerpunkte wünschen. Einen gewichtigen Anteil hieran machen Kindergärten aus, in denen neben dem Deutschen noch eine oder mehrere andere Sprachen gelehrt bzw. gefördert werden (vgl. Pfaff et al. 2014 für einen Überblick zu Angeboten in Berlin und Brandenburg; Meier 2010 mit einem Forschungsüberblick zu den sozialen und sprachlichen Effekten mehrsprachiger Kinderbetreuung). Der Staat unterstützt solche Initiativen in der Regel durch Anerkennung der Einrichtungen und unter Umständen auch durch Finanzierungen. Die Gründung selbst geht allerdings häufig zurück auf eine Lücke, die durch staatliche Angebote nicht gefüllt wird und deshalb erst auf Basis von Eigeninitiativen abgedeckt werden kann. Damit verbunden sind oft Zugangsbarrieren insbesondere durch Beiträge oder Gebühren, die weniger privilegierte Familien ausschließen (Wode 2016: 21, zum Zusammenhang zwischen sozioökonomischem Status und Kinderbetreuung vgl. auch Kreyenfeld/Krapf 2010).

Die Sprachkombinationen, die im Kita-Bereich in Berlin vorzufinden sind, sind so vielfältig wie die Sprachlandschaft der Stadt. Deutschlandweit stand Berlin 2014 im Hinblick auf die absolute Anzahl bilingualer Kinderbetreuungseinrichtungen auf dem zweiten Platz nach dem Saarland (FMKS 2014a: 7). Die öffentlich-private Agentur Berlin Partner für Wirtschaft und Technologie erstellt in regelmäßigen Abständen einen Überblick der Vorteile von Berlin als Wirtschaftsstandort. Hierbei werden unter anderem auch mehrsprachige Kinderbetreuungsangebote aufgelistet, um internationale bzw. mehrsprachige Arbeitnehmer^innen und Investor ${ }^{\star}$ innen von einer Ansiedelung in Berlin zu überzeugen. ${ }^{19}$ Bereits mit diesem relativ einfachen Service zeigt sich, wie auch die mehrsprachige

$19 \mathrm{https} / /$ staging.businesslocationcenter.de/informationen-fuer-neu-berliner/tagesbetreu ung-von-kindern/mehrsprachige-kitas/. 
Kinderbetreuung eine ökonomische Dimension bekommen kann, wenn sie als Standortvorteil geltend gemacht wird. Auch der Berliner Senat bietet die Möglichkeit an, in seinem Kita-Verzeichnis gezielt Betreuungseinrichtungen mit bestimmten Sprachschwerpunkten zu suchen. ${ }^{20}$ Vergleicht man die am häufigsten angebotenen Sprachen in Kitas, die bilingual oder multilingual arbeiten, so sind wenig überraschend englisch-deutsche Kitas deutlich in der Mehrheit. Etwa gleichauf dahinter liegen Kindertagesstätten, die Betreuung auf Türkisch, Spanisch oder Russisch anbieten (Tab. 5.1).

Tab. 5.1: Anzahl von Kindertagesstätten in Berlin, die neben dem Deutschen noch eine oder mehrere andere Sprachen anbieten (Februar 2019).

\begin{tabular}{lr}
\hline Sprache & Anzahl Einrichtungen \\
\hline Englisch & 157 \\
\hline Türkisch & 50 \\
\hline Spanisch & 48 \\
\hline Russisch & 47 \\
\hline Arabisch & 32 \\
\hline Französisch & 31 \\
\hline Italienisch & 23 \\
\hline
\end{tabular}

Kitas, die mehrere Sprachen anbieten, sind in der Tabelle für die entsprechenden Sprachen jeweils mitgezählt. Bei Trägern von Einrichtungen an mehreren Standorten wurde jeder Standort einzeln gezählt.

Auffällig ist, dass das Französische vom Spanischen deutlich übertroffen wird, sowohl in der Anzahl der Einrichtungen als auch der Betreuungsplätze (Spanisch: 1446 Betreuungsplätze im Jahr 2017, Französisch 811). ${ }^{21}$ Deutschlandweit steht nach dem Englischen das Französische dagegen klar an zweiter Stelle in der Kita-Anzahl, insbesondere durch den starken Ausbau in den Grenzregionen

20 https://www.berlin.de/sen/jugend/familie-und-kinder/kindertagesbetreuung/kitas/ver zeichnis/.

21 Die Anzahl der Betreuungsplätze wurde durch eine Erhebung anhand der Webseiten der bilingualen Betreuungseinrichtungen (deutsch-spanisch, deutsch-französisch) im September 2017 ermittelt. Die Zahlen können sich jedoch durch die starke Fluktuation der Angebote bereits nach kurzer Zeit deutlich verändert haben. 
im Saarland und in Rheinland-Pfalz. Danach folgt Dänisch aufgrund der intensiven Förderung in Schleswig-Holstein, und Spanisch steht deutschlandweit an vierter Stelle (FMKS 2014a: 1).

In den allermeisten Fällen werden in den mehrsprachigen Kindergärten Berlins alle Altersstufen abgedeckt, von der Krippe für Kleinkinder bis hin zur Vorschule mit einer Hinführung zum Übergang in die Grundschule. Dabei ist in aller Regel die Erziehung tatsächlich zweisprachig, d. h. eine Kombination aus Deutsch mit einer weiteren Sprache. Angebote mit mehr als zwei Sprachen sind selten. Einige größere Einrichtungen bieten eine Auswahl aus mehreren Sprachen, wobei die Kinder dann jedoch wiederum in einzelne zweisprachigen Gruppen aufgeteilt sind.

Neben den weiter verbreiteten Sprachen können Eltern in Berlin auch bilinguale Kitas mit seltener angebotenen Sprachkombinationen finden, zum Beispiel solche mit mehrsprachiger Erziehung in Kombination mit Griechisch, Vietnamesisch oder Hebräisch. Das Spanische gehört in diesem Bereich also längst nicht mehr zu den ungewöhnlichen Angeboten.

Welche Motivationen und Erwartungen haben Eltern, die ihre Kinder in einer spanisch-deutschen zweisprachigen Kita anmelden? Diese Frage war Gegenstand einer Untersuchung mit freundlicher Beteiligung zweier bilingualer Einrichtungen aus Berlin, einer kleineren Kita in Neukölln (22 Betreuungsplätze) und einer etwas größeren in Charlottenburg (140 Plätze). Beide Kitas arbeiten nach der Immersionsmethode, d. h. muttersprachliche Fachkräfte betreuen die Kinder durchgehend in der jeweils eigenen Erstsprache, so dass alle täglich in großem Umfang sowohl mit dem Spanischen als auch mit dem Deutschen in Berührung kommen.

Nach einer Projektvorstellung in den Kitas wurden Eltern gebeten, einen Online-Fragebogen zum Familienhintergrund, zu den sprachlichen Praktiken und Haltungen und zu den Hintergründen der Kita-Wahl auszufüllen. Neben einigen Angaben zu demographischen Daten war der Fragebogen größtenteils qualitativ ausgelegt, so dass die Befragten offene Fragen mit Freitextkommentaren beantworten konnten. Damit wurde vermieden, den jeweils sehr unterschiedlichen familiären Konstellationen ein vorgegebenes Schema überzustülpen. An beiden Standorten wurden jeweils 14 Elternteile befragt, die zudem Informationen auch zu ihren Partner*innen angaben. ${ }^{22}$ Die genauen familiären Verhältnisse wurden dabei nicht abgefragt; es blieb den Teilnehmerinnen selbst überlassen zu

22 Die Untersuchung war Teil eines studentischen Forschungsprojekts im Rahmen eines Seminars im Soziolinguistik-Modul des MA-Studiengangs Sprachwissenschaft an der Freien Universität Berlin. Konzipiert und durchgeführt wurde die Datensammlung von Nina Brenner, Celina Büscher, Sabrina Döbel, Friederike Fischer, Franziska Heydemann, Lara Kast, Elzem Kaya, Alina Klima, Vera Link und Britta Stuhl. 
entscheiden, wen sie als Mitglieder der Kernfamilie, Partner*in oder Vater bzw. Mutter der Kinder ansehen. ${ }^{23}$ Die meisten Befragten waren in akademischen Berufen tätig, einige wenige gaben Berufe mit anderen Qualifikationen an.

Die große Mehrheit der Befragten (24 Personen) hatte die deutsche Staatsbürgerschaft, die spanische (2), kubanische (1) und chilenische (1) waren ebenfalls vertreten (Tab. 5.2). Doppelte Staatsbürgerschaften waren nicht vorhanden. Die meisten gaben an, in Deutschland geboren $\mathrm{zu}$ sein oder seit vielen Jahren in Deutschland zu leben. Es wurden jedoch von deutlich mehr Befragten weitere hispanophone Länder genannt, zu denen sie selbst oder ihre Partner*innen eine persönliche Bindung haben (als ,Herkunftsland'), auch wenn sie nicht deren Staatsbürgerschaft besitzen. An erster Stelle stand hier Spanien, aber auch einige lateinamerikanische Länder wurden genannt.

Tab. 5.2: Herkunftsländer, die von den Befragten genannt wurden (eigene oder Partner*in; Mehrfachnennungen möglich).

\begin{tabular}{lr}
\hline Herkunftsland & Anzahl Nennungen \\
\hline Deutschland & 35 \\
\hline Spanien & 7 \\
\hline Argentinien & 3 \\
\hline Chile & 2 \\
\hline Costa Rica & 1 \\
\hline Kolumbien & 1 \\
\hline Kuba & 1 \\
\hline anderes nicht hispanoph. Land & 1 \\
\hline
\end{tabular}

Der Überblick zeigt, dass für die übergroße Mehrheit Deutschland als Herkunftsland gesehen wird. Die Bedeutung des Begriffs wurde bewusst offen gelassen um alle möglichen persönlichen Bezüge einbeziehen zu können. Aus der Anzahl der Nennungen ergibt sich, dass für viele Befragte und ihre Partner*innen mehrere Länder als Herkunfts- bzw. Bezugsländer angesehen werden. Dabei halten sich Spanien und lateinamerikanische Länder fast exakt die Waage. Obwohl Spanien

23 Alleinerziehende oder Familien mit mehr als zwei elterlichen Bezugspersonen waren nicht ausgeschlossen, aber alle Befragten gaben an, in Familienkonstellationen mit zwei Elternteilen zu leben. 
stark vertreten ist, kann es deshalb nicht als einziges oder auch nur dominantes Bezugsland der Eltern gelten.

Auch bei den Angaben zur eigenen Muttersprache bzw. derjenigen der Partner^innen überwog das Deutsche, wobei das Spanische stark präsent war (Tab. 5.3).

Tab. 5.3: Angaben der Befragten zu ihren eigenen Muttersprachen und denen ihrer Partner^innen (Mehrfachnennung möglich).

\begin{tabular}{lrr}
\hline Sprache & eigene & Partner/in \\
\hline Deutsch & 23 & 18 \\
\hline Spanisch & 6 & 10 \\
\hline andere & - & 3 \\
\hline
\end{tabular}

Auch hier ergibt sich aus der Anzahl der Antworten, dass zahlreiche Befragte mehrsprachig waren und sowohl Deutsch als auch Spanisch als Muttersprachen ansahen. Diejenigen, bei denen das nicht der Fall war, gaben zum allergrößten Teil an, die jeweils andere Sprache dennoch gut zu beherrschen. Es scheint demnach bei den Befragten eine starke sprachliche Symmetrie zu geben, so dass in den Beziehungen jeweils beide Elternteile auch die Sprache des/der Anderen angenommen haben. Möglicherweise ist bereits dies ein Indiz für eine generelle Affinität zur Mehrsprachigkeit und für ein Interesse an sprachlicher Diversität, was auch einen Einfluss auf die Wahl der Kinderbetreuungsstätte haben kann.

Allerdings wurde von einigen Befragten auch angegeben, dass sie selbst in ihrer Familie gar keinen Bezug zum Spanischen hatten. Nach den Beweggründen für die Wahl der Kita gefragt, wurde mehrfach wiederkehrend gesagt, dass in anderen Einrichtungen kein Platz frei war oder dass die gewählte Kita schlichtweg günstig zum Wohnort lag. Rein praktische Gründe können demnach durchaus den Ausschlag geben, ohne dass tiefgreifende Überlegungen zur sprachlichen Entwicklung des Kindes an erster Stelle stehen. Unter Umständen könnte dies in der Ansiedlungspolitik von Kindertagesstätten neue Impulse liefern, denn offenbar bekommen auch Eltern mit einsprachigem Hintergrund durch die reine Verfügbarkeit bereits einen Anreiz, für ihre Kinder eine mehrsprachige Kita zu wählen. Auch wenn die mehrsprachige Erziehung nicht das entscheidende Kriterium war, wird sie dennoch von den Eltern geschätzt. So schreibt ein*e Teilnehmerin:

Für uns stand nicht die Sprache im Vordergrund, sondern die sehr positiven Empfehlungen zu dieser Kita [. . . ]. Der zusätzliche Spracherwerb war ein positiver Nebeneffekt, den wir jetzt allerdings so schnell auch nicht wieder verlieren wollen. 
Bei den Eltern, die für ihr Kind bewusst eine spanisch-deutsche bilinguale Kita gesucht haben, überwiegt eine eher abstrakte und eine konkrete Zielsetzung. Auf abstrakter Ebene wird die zweisprachige Kinderbetreuung als Zugang zu einem anderen Kulturbereich gesehen, mit dem das Kind in Berührung kommen soll. Hier wird sowohl die „lateinamerikanische Kultur“ als auch die „spanische Kultur“ genannt. Was genau darunter verstanden wird, bleibt an dieser Stelle offen. Klar ist jedoch, dass die Kita demnach nicht nur als zweisprachig sondern auch als multikulturell aufgefasst wird. Die Eltern erwarten also nicht nur sprachliche Förderung, sondern auch zusätzlichen Input, mit denen die Kinder mit kulturellen Codes vertraut gemacht werden, die über die Sprachverwendung an sich hinausgehen.

Als konkretes Ziel formulieren einige Eltern, dass die Kinder die Kommunikationsfähigkeit mit spanischsprachigen Familienmitgliedern nicht verlieren sollen. Beispielsweise schreibt ein*e Teilnehmer*in, man habe sich für die bilinguale Kita entschieden, damit „es [das Kind] viel Spanisch spricht und gut mit seiner spanischen Familie kommunizieren kann.“ Der Kita-Besuch soll also auch einen Nutzen haben, der sich unmittelbar im Familienumfeld niederschlägt. Dabei zeigt sich, dass die Befragten der Kinderbetreuung eine wichtige Rolle dabei zuweisen, das mehrsprachige Familienumfeld mit (zumeist) einem deutsch- und einem spanischsprachigen Anteil zusammenzuhalten. So geben etwa mehrere Befragte an, die Wahl sei auch deshalb auf die zweisprachige Einrichtung gefallen, damit der/die Partner*in in den Kita-Alltag eingebunden sein kann und nicht nur ein Elternteil die Verbindung mit dem Personal und anderen Eltern übernimmt.

Da die zweisprachigen Kindergärten ein ausgearbeitetes Konzept zur Sprachverwendung und -förderung haben, können Eltern diese in ein Verhältnis zu den eigenen sprachlichen Praktiken in der Familie setzen. So schreibt jemand zu den Gründen für die Kita-Wahl: „Fortführung der spanischen Sprache in der Familie. Spanisch soll mehr gefördert werden, da Familiensprache Deutsch ist.“

In diesem Zusammenhang ist es interessant, einen genaueren Blick auf den Umgang der befragten Familien mit der Mehrsprachigkeit im Privatleben zu werfen. Dazu wurden die Befragten gebeten, nähere Angaben zur Nutzung der verschiedenen Sprachen im Familienkontext zu machen, und zwar zwischen den Elternteilen, mit den Kindern und, falls mehrere Kinder im Haushalt leben, der Kinder untereinander. Es zeigt sich, dass viele Eltern das Prinzip „eine Person eine Sprache“ anzuwenden versuchen, wobei keine genaueren Angaben gemacht wurden, wie konsequent dies gelingt. In vielen Fällen wurde jedenfalls pro Elternteil in der Kommunikation mit den Kindern nur eine einzige Sprache angegeben. Es bestätigte sich zudem die Beobachtung vieler mehrsprachiger Familien, dass die Kinder untereinander meist die im Umfeld dominantere Sprache nutzen, in diesem Fall also eher Deutsch als Spanisch. Am meisten variierte der Sprachgebrauch der Eltern untereinander. Hier waren trotz der vergleichsweise kleinen Anzahl an 
Befragten alle denkbaren Kombinationen vorhanden, von der ausschließlichen Nutzung des Deutschen oder Spanischen über eine Tendenz zugunsten einer der beiden Sprachen bis hin zur (nach Selbstwahrnehmung) unsystematischen Abwechslung beider Sprachen.

Die Qualität bzw. die Quellen des Inputs im Spanischen unterscheiden sich ebenso stark wie die sprachlichen Praktiken in den Familien. Neben Gesprächen mit den spanischsprachigen Elternteilen oder anderen Familienmitgliedern, zum Teil auch auf Distanz per Telefon oder Skype u.ä., fällt insbesondere der gezielte Einsatz von Medien ins Auge. Sehr viele Befragte geben an, ihren Kindern Filme und DVDs, Musik oder Bücher auf Spanisch zur Verfügung zu stellen oder ihnen vorzulesen. Einige Befragte gaben jedoch an, zuhause werde kaum oder gar kein Spanisch genutzt - dies liegt logischerweise nahe bei den Familien, in denen keines der Elternteile Spanisch spricht.

Die sprachliche Entwicklung der Kinder bewerteten die Befragten durchweg positiv. Viele wiesen darauf hin, dass das eigene Kind im Spanischen vor allem passive Kenntnisse habe und selbst eher Deutsch spreche. Dennoch äußerte sich niemand unzufrieden; die meisten setzten die Entwicklung in Bezug zu realistischen Erwartungen im bilingualen Spracherwerb und scheinen sich der Tatsache bewusst zu sein, dass die verschiedenen Sprachen für die Kinder je nach Altersund Lebensphase unterschiedliche Rollen spielen können. Dazu gehört auch, in bestimmten Sprachen zunächst vor allem Fortschritte im Verstehen zu machen, bevor sie auch aktiv genutzt werden (Wode 2016: 28f., 2006: 15).

Angesichts der enorm unterschiedlichen sprachlichen Praktiken und damit auch einer großen Bandbreite des sprachlichen Inputs für die Kinder stellt die gesteuerte und geplante Sprachförderung in der Kita eine wichtige Konstante dar, auf die sich die Eltern verlassen. Insgesamt deuten die Antworten auf ein sehr hohes Sprachbewusstsein der Eltern hin, auch bei denjenigen, die zuhause einsprachig deutsch kommunizieren. Für die Kinder dürfte dies einen doppelten Vorteil mit sich bringen: Sie erhalten durch die Sprachkonzepte der Kitas zusätzliche sprachliche Förderung, werden zugleich aber auch in der Familie beeinflusst durch Bezugspersonen, die über Sprache ausführlich nachdenken und sich informieren.

Für die befragten Familien erfüllen die zweisprachigen Kitas eine doppelte Funktion, nämlich eine der Überdachung und eine der Ergänzung. Sie überdachen insbesondere die verschiedensprachigen Familienzweige und sorgen dafür, dass die Kinder durch den Aufenthalt in einer ansonsten dominant deutschsprachigen Gesellschaft nicht die Verbindung zu spanischsprachigen Familienmitgliedern verlieren. Zudem überdacht die Kita den multikulturellen Hintergrund der Kinder und sorgt durch die Vermittlung spezifischer kultureller Codes für eine Art abstrakte familienhistorische Kontinuität. 
Die Ergänzungsfunktion der Kitas ergibt sich aus dem sprachlichen Input der nicht-dominanten Sprache bzw. überhaupt dem Input einer im Familienkontext nicht vorhandenen Sprache. Da das gesellschaftliche Umfeld deutlich überwiegend deutschsprachig geprägt ist, kommt der Kita die Aufgabe zu, den Kontakt zu den beiden Sprachen auszubalancieren. Dies ist insbesondere denjenigen Eltern wichtig, die diese Balance in ihrem Familienumfeld nicht selbst herstellen können.

Die tiefgreifenden Reflexionen der Eltern zeigen auf, dass sie die sprachliche Ausrichtung der Kita als ersten Schritt sehen, auf dem der weitere Spracherwerbsgang der Kinder aufbauen soll. Die Überlegungen zur Wahl der Kinderbetreuung sind stets mit einer zumindest groben Zukunftsperspektive verknüpft, nämlich den Kindern möglichst gute Voraussetzungen für den weiteren Lebens- und auch Bildungsweg zu geben. Dass das Spanische Teil dieses Weges sein wird, scheinen die meisten Eltern als wahrscheinlich anzusehen. Dementsprechend wichtig ist für sie sicherlich die Frage, welche Rolle das Spanische in den folgenden Stufen des Bildungssystems spielt.

\subsection{Grundschule}

In der Grundschule bleibt in Berlin die Zahl der vorhandenen Plätze für zweisprachige deutsch-spanische Bildung etwa stabil. Zwei Schulen unterrichten im Rahmen des Europaschulen-Verbunds etwa 1350 Schülerinnen und Schüler in den beiden Sprachen als Unterrichtsmedium, was im Jahr 2017 ungefähr dem Platzangebot an multilingualen Kindergärten mit Spanischangebot entsprach (s. oben). Der Berliner Senat kündigte 2016 nach der Wahl zum Abgeordnetenhaus an, je eine weitere deutsch-spanische zweisprachige Schule im Primar- und im Sekundarbereich einzurichten. Die dritte bilinguale Grundschule hat zum Beginn des Schuljahres 2018/19 den Unterrichtsbetrieb als Zweig der Europa-Schule aufgenommen. Dies deutet auf eine wachsende Nachfrage hin, aber auch auf eine Reaktion der Politik auf die Bedürfnisse einer zunehmend mehrsprachigen Bevölkerung. Zum gleichen Zeitpunkt wurde beispielsweise angekündigt, auch mehr bilinguale deutsch-arabische Angebote zu schaffen.

Deutschlandweit sind deutsch-spanische bilinguale Grundschulen noch relativ selten. 2014 stand das Spanische an siebter Stelle in der Anzahl von Grundschulen in Deutschland, die im Rahmen von Content and Language Integrated Learning (CLIL) ${ }^{24}$ eine weitere Sprache neben dem Deutschen vermittelten. Nach

24 Entsprechend dem CLIL-Konzept wird eine Sprache nicht nur als einzelnes Fach unterrichtet, sondern auch andere Schulfächer bzw. Lerninhalte durch die Sprache als Unterrichtsme- 
den bereits im Kita-Bereich deutlich stärker vertretenen Sprachen Englisch, Französisch und Dänisch sind dies bei den Grundschulen außerdem Sorbisch, Italienisch und Griechisch. Dabei muss jedoch berücksichtigt werden, dass einige dieser Sprachen ähnlich wie im Bereich der Kindergärten stark regional konzentriert sind (etwa Griechisch in Bayern, Sorbisch in Sachsen), während Spanisch und auch Italienisch weiter gestreut sind (FMKS 2014b). Berlin zeichnet sich sowohl bei Kitas als auch bei Grundschulen dagegen durch eine große Sprachenvielfalt aus.

In den frühen Phasen des Bildungssystems, von der Kita bis zur Grundschule, ist das spanischsprachige Bildungsangebot stark auf Zweisprachigkeit ausgerichtet, meist mit Immersionskonzepten. Spanisch wird hier also als Kommunikationsmedium in der Kitabetreuung bzw. im Grundschulunterricht eingesetzt. Zielgruppe solcher Einrichtungen sind einerseits Kinder von Eltern mit einem spanischsprachigen Hintergrund (eines Elternteils oder der gesamten Familie), andererseits auch Kinder aus nicht-spanischsprachigen Familien, für die eine mehrsprachige Erziehung attraktiv erscheint. Rasche (2014) sieht bei der Auswahl bilingualer Grundschulen vor allem das Streben der Eltern nach dem Erwerb von „transnationalem Humankapital“ (vgl. auch Pomerantz 2002 für ähnliche Überlegungen bei Studierenden in den USA). Gemeint ist damit, dass die frühe Mehrsprachigkeit den Kindern in der Zukunft soziale, kommunikative und auch räumliche Mobilität eröffnet. Rasches Untersuchung zu den Motivationen zur Wahl bilingualer Grundschulen konzentriert sich auf deutsch-englische Angebote, während als ,typisch migrantisch` angesehene Sprachen wie Türkisch oder Arabisch gar nicht berücksichtigt wurden. Das Englische wird sicher bei vielen einsprachig deutschen Familien tatsächlich als besonders starke Ressource für die Zukunft der Kinder angesehen. Man kann vermuten, dass die relativ instrumentalistische Herangehensweise beim Spanischen etwas weniger ausgeprägt ist, wie es die oben genannten Beispiele im Bereich bilingualer Kindergärten bereits andeuten.

Auch wenn es für die Herangehensweise an bilinguale Erziehung bzw. Bildung ein wichtiger Faktor ist, ob die zweite Sprache auch in der Familie bereits vorhanden ist, lässt sich angesichts des jungen Alters der Kinder, gerade bei Angeboten von Kinderkrippen für die Jüngsten, selten sinnvoll zwischen Kindern mit Spanisch als ,Muttersprache“ und als ,Zweit-“ oder gar ,Fremdsprache“ unterscheiden (Wode 2006: 5). Der Unterschied liegt hauptsächlich im Input der vorhandenen Sprachen, der teils neben der Kita oder Schule auch aus der Familie kommt, während bei anderen Kindern die Bildungseinrichtung und

dium vermittelt. Der Umfang des Fachunterrichts in der Sprache kann dabei unterschiedlich groß sein. 
ggf. der dort aufgebaute Freundeskreis der einzige Berührungspunkt mit der anderen Sprache ist. Ziel solcher mehrsprachiger Einrichtungen ist es, durch den frühen Kontakt mit der Sprache schon Kenntnisse zu vermitteln, bevor überhaupt von ,muttersprachlichem Unterricht' oder ,Fremdspracherwerb“ die Rede sein kann. Selbst im Grundschulbereich ist es häufig noch nicht sinnvoll, hier eine scharfe konzeptuelle und methodische Trennlinie zu schaffen. In den allermeisten Fällen erfolgt die Sprachvermittlung bzw. -förderung allerdings durch gezielten Fachunterricht und nicht unbedingt im Rahmen der Nutzung als Unterrichtsmedium, wenn auch in der Regel mit stärker impliziten, altersgerechten Methoden der Didaktik.

Als Fachunterricht ohne Vorkenntnisse außerhalb integraler bilingualer Konzepte wird an Berliner Grundschulen in der Regel nur Englisch und Französisch angeboten. Spanisch als Fremdsprache vermittelt eine kleine Anzahl privater bzw. freier Grundschulen. Was das Spanische betrifft, ändert sich die Ausrichtung der Sprachvermittlung und damit auch die Art des Sprachkontakts und -erwerbs deutlich mit dem Übergang zum Sekundarbereich, weil erst dort klassischer Fremdsprachenunterricht eingeführt wird.

\subsection{Weiterführende Schulen}

Zweisprachiger deutsch-spanischer Unterricht mit Immersionsmethode ist in der Berliner Sekundarstufe selten. Lange Zeit gab es nur eine Integrierte Sekundarschule als Teil des Netzwerks der Europaschulen mit Schwerpunkt Spanisch, eine zweite kam zum Schuljahr 2017/18 hinzu. Damit liegt die Anzahl der Schüler^innen in bilingualen deutsch-spanischen Sekundarschulen bei etwa 1350 und damit auf dem gleichen Niveau wie bei mehrsprachigen Kindergärten und Grundschulen.

Eine quantitativ weitaus größere Bedeutung hat das Spanische in der Sekundarstufe hingegen als Fremdsprache. Hier trennt sich aufgrund des schon etwas fortgeschrittenen Alters der Schüler*innen das Konzept der Sprachvermittlung in Angebote für diejenigen, die bereits umfangreiche Vorkenntnisse mitbringen (aus bilingualen Kitas und Grundschulen, durch familiäre Hintergründe oder Zuzug aus dem spanischsprachigen Ausland), und in die klassische Fremdsprachenlehre ohne Vorkenntnisse.

Spanisch als Fremdsprache ist in der Sekundarstufe in Berlin längst etabliert und weit verbreitet. Damit spiegelt Berlin eine deutschlandweite Entwicklung wider: Bereits in den vergangenen Jahrzehnten war Spanisch in den meisten Bundesländern die am dritthäufigsten gewählte Fremdsprache, und von 2002 bis 2004 stieg deutschlandweit die Anzahl der Spanischlernenden im allgemeinen Schulwesen um über 25\% an, in Berlin sogar um über 50\% 
(Ministerio de educación y ciencia 2005: 17, 25). Bereits 2005 stellte das spanische Bildungsministerium mit Blick auf die Situation in Deutschland fest, „que la demanda real es mayor de lo que las cifras representan, y que no se ve satisfecha por diferentes factores, como la fuerte presión que ejerce el profesorado de francés para que se mantenga la enseñanza de este idioma [...]“ (Ministerio de educación y ciencia 2005, 24, vgl. auch S. 17).

In den zehn Jahren zwischen den Schuljahren 2004/05 und 2014/15 hat sich in der Bundesrepublik die Anzahl der Spanischlernenden an Schulen nahezu verdoppelt (Malecki 2016: 21). In jüngster Zeit stieg die Anzahl der Spanischlernenden an allgemeinen und berufsbildenden Schulen innerhalb nur eines Schuljahrs um 4,4\%, während die Anzahl der Französischlernenden um 2,7\% sank. ${ }^{25}$ Bär (2017) weist zu Recht darauf hin, dass die Vergleichszahlen beider Sprachen nicht ohne Weiteres in ein direktes Verhältnis zueinander gesetzt werden können, das die sichtbare Zunahme des Interesses am Spanischen unmittelbar mit einem Beliebtheitsverlust des Französischen erklären soll.

Bei Spanisch als Schulfremdsprache sind insbesondere die Stadtstaaten die Vorreiter. In Hamburg und Bremen ist Spanisch bereits die am zweithäufigsten gelernte Fremdsprache. In Berlin steht es aktuell an dritter Stelle nach Französisch und wird von etwa 8\% der Schülerinnen und Schüler belegt. Deutschlandweit sind dies gut 5\% (Hoffmann/Malecki 2018). Generell gelten Spanisch wie auch Französisch und Italienisch als typische zweite oder dritte Fremdsprachen in den Bildungssystemen Deutschlands (Bär 2017: 86). Für Berlin wird diese Abfolge unten weiter aufgeschlüsselt.

Diese Zahlen schreiben die Haltungen fort, die bereits bei einer Befragung des Instituts für Deutsche Sprache in Mannheim aus dem Jahr 2008 festgestellt wurden. Von etwa 2000 Befragten sagten 95,5\%, dass Englisch in den Schulen unterrichtet werden sollte, 65,8\% waren beim Französischen dieser Meinung und 39,3\% beim Spanischen (Gärtig/Plewnia/Rothe 2010: 250).

Von 284 weiterführenden Schulen in Berlin boten zum Schuljahr 2016/17 gut die Hälfte (139 Schulen, ca. 48,9\%) Spanisch als Fremdsprache an. ${ }^{26}$ Vor 2005 lag die Anzahl der weiterführenden Schulen in Berlin, die Spanisch als Fremdsprache anboten, noch bei ungefähr 50 (Ministerio de educación y ciencia 2005: 19). Der heutige Wert ist nahezu identisch bei öffentlichen Schulen und solchen

25 Angaben des Statistischen Bundesamts für die Schuljahre 2017/18 und 2018/19: https:// www.destatis.de/DE/Themen/Gesellschaft-Umwelt/Bildung-Forschung-Kultur/Schulen/Tabel len/allgemeinbildende-beruflicheschulen-fremdsprachl-unterricht.html.

26 Alle Angaben zu weiterführenden Schulen in Berlin gelten für das Schuljahr 2016/17 entsprechend dem Schulwegweiser der Senatsverwaltung für Bildung, Jugend und Wissenschaft. 
in freier Trägerschaft. Kaum Unterschiede im Spanischangebot zeichnen sich ab bei einer Aufschlüsselung nach geographischer Lage in der Stadt und Schulform.

Die räumliche Verteilung des Spanisch-Angebots an weiterführenden Schulen in Berlin stellt sich sehr gleichmäßig dar. Es gibt weder ein Übergewicht zugunsten innerstädtischer Schulen noch zugunsten von Schulen im ehemaligen Westteil der Stadt (Tab. 5.4, 5.5). Dies wäre durchaus erwartbar gewesen, wenn Spanisch als besonderes Merkmal zu betrachten wäre, das beispielsweise von Schüler^innen aus sozial privilegierten Familien häufiger nachgefragt wird. Da sowohl in den zentralen Bezirken als auch am Stadtrand sowohl im Westen als auch im Osten Familien mit hohem Einkommen und Bildungsstand anzutreffen sind, ist die Aufteilung nach diesen beiden Kriterien vermutlich zu grob. Etwas mehr Aufschluss gibt hier die Aufschlüsselung nach Schultypen (Tab. 5.6).

Tab. 5.4: Anzahl der Schulen, die Spanisch als Fremdsprache anbieten, nach Lage in Berlin (innerhalb oder außerhalb des S-Bahn-Rings).

\begin{tabular}{lrr}
\hline & mit Spanisch & ohne Spanisch \\
\hline innerhalb S-Bahn-Ring & 36 & 37 \\
\hline außerhalb S-Bahn-Ring & 103 & 108 \\
\hline
\end{tabular}

Tab. 5.5: Anzahl der Schulen, die Spanisch als Fremdsprache anbieten, nach Lage in Berlin (ehemaliger Westteil und Ostteil der Stadt).

\begin{tabular}{lrr}
\hline & mit Spanisch & ohne Spanisch \\
\hline Ost & 56 & 56 \\
\hline West & 83 & 89 \\
\hline
\end{tabular}

Tab. 5.6: Spanisch als Fremdsprache an Berliner Schulen, aufgeschlüsselt nach Schulformen.

\begin{tabular}{lcrr}
\hline Schulform & Gesamtzahl & $\begin{array}{r}\text { Anzahl Schulen mit } \\
\text { Spanisch als FS }\end{array}$ & $\begin{array}{r}\text { Anteil Schulen mit } \\
\text { Spanisch als FS }\end{array}$ \\
\hline $\begin{array}{l}\text { Integrierte } \\
\text { Sekundarschule }\end{array}$ & 121 & 56 & $46,3 \%$ \\
\hline Gymnasium & 114 & 60 & $52,6 \%$ \\
\hline Gemeinschaftsschule & 36 & 20 & $55,6 \%$ \\
\hline Waldorfschule & 10 & 2 & $20 \%$ \\
\hline Eliteschule des Sports & 3 & 1 & $33,3 \%$ \\
\hline
\end{tabular}


Daten aus dem ersten Jahrzehnt des 21. Jahrhunderts zeigen, dass deutschlandweit das Spanische vor allem in Gymnasien als Fremdsprachenfach verbreitet war, deutlich seltener dagegen wurde es an Gesamt- oder Realschulen angeboten (Klump/Willems 2012: 165). Auch in Berlin fällt ins Auge, dass Spanisch als Fremdsprache überdurchschnittlich oft an Gemeinschaftsschulen und Gymnasien angeboten wird. Bei den Integrierten Sekundarschulen ${ }^{27}$ ist der Anteil leicht unterdurchschnittlich. Der Unterschied ist nicht drastisch, dennoch scheint der Vergleich darauf hinzudeuten, dass Spanisch etwas stärker als Qualifikation der sozial stärkeren Schichten gesehen werden kann, deren Kinder weiterhin häufiger Gymnasien besuchen. Bei dieser Beobachtung muss berücksichtigt werden, dass an Gymnasien ohnehin eine höhere Zahl von Fremdsprachen angeboten werden wird (Tab. 5.7).

Tab. 5.7: Anzahl der insgesamt angebotenen Fremdsprachen nach Schulformen an weiterführenden Schulen in Berlin.

\begin{tabular}{lrccr}
\hline & \multicolumn{4}{c}{ Anzahl Fremdsprachen } \\
\cline { 2 - 5 } Schulform & $\mathbf{2}$ & $\mathbf{3}$ & $\mathbf{4}$ & $\mathbf{5}$ \\
\hline Gemeinschaftsschule & 15 & 15 & 4 & 1 \\
\hline Gymnasium & 5 & 28 & 59 & 17 \\
\hline Integrierte Sekundarschule & 54 & 47 & 15 & 5 \\
\hline
\end{tabular}

Das Distinktionsmerkmal, das insbesondere Gymnasien prägt, ist also ein generell umfangreicheres Fremdsprachenangebot, zu dem das Spanische zählt. Dies ist auch bundesweit zu beobachten (Bär 2017: 89). Während die überwiegende Mehrheit der Integrierten Sekundarschulen zwei oder drei Fremdsprachen anbietet, sind vier oder mehr Fremdsprachen an Gymnasien praktisch die Regel. Es überrascht wenig, dass in diesen Fällen die vergleichsweise beliebte Fremdsprache Spanisch mit dabei ist. Je höher die Anzahl der angebotenen Fremdsprachen ist, desto größer ist der Anteil der Schulen, die unter diesen Sprachen auch Spanisch anbieten. Dies gilt für die Mehrheit der weiterführenden Schulen (56,8\%), die nur drei Fremdsprachen anbieten (Abb. 5.1). Hier hat das Spanische bereits begonnen, das Latein zu verdrängen, das an dieser Stelle zu erwarten gewesen wäre: Wenn eine Schule drei Fremdsprachen anbietet, ist stets

27 Die Integrierte Sekundarschule führt in Berlin die früheren Haupt-, Real- und Gesamtschulen zusammen und bietet Schulabschlüsse der verschiedenen Stufen bis zum Abitur an. 


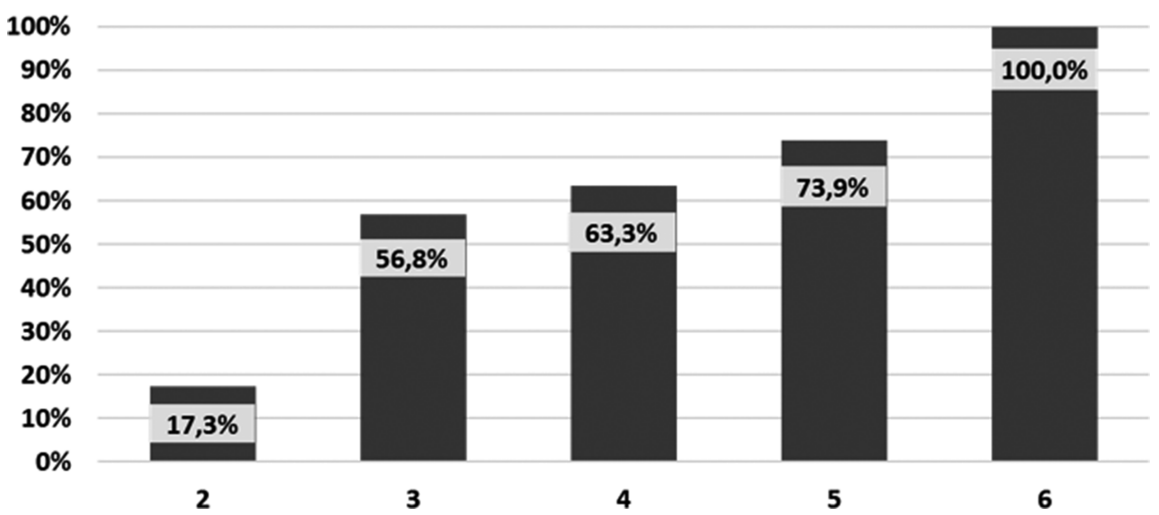

Abb. 5.1: Anteil der weiterführenden Schulen in Berlin (in Prozent), die Spanisch als Fremdsprache anbieten, abhängig von der Gesamtzahl an angebotenen Sprachen.

Englisch mit dabei und Französisch die deutlich am weitesten verbreitete weitere Sprache. Den dritten Platz nimmt dann mehrheitlich das Spanische ein.

Diese Entwicklung zeigt sich auch daran, dass Spanisch in der Sprachenfolge der Sekundarschulen meist relativ weit vorn steht (Abb. 5.2). Etwa 82\% der Schulen (114 von 139), in denen Spanisch gelernt werden kann, bieten es bereits als mögliche zweite Fremdsprache an. Häufig kann es an derselben Schule auch später noch belegt werden. Dass Spanisch generell als zweite Sprache gewählt werden kann, in der Regel nach Englisch, deutet auf einen hohen Stellenwert im

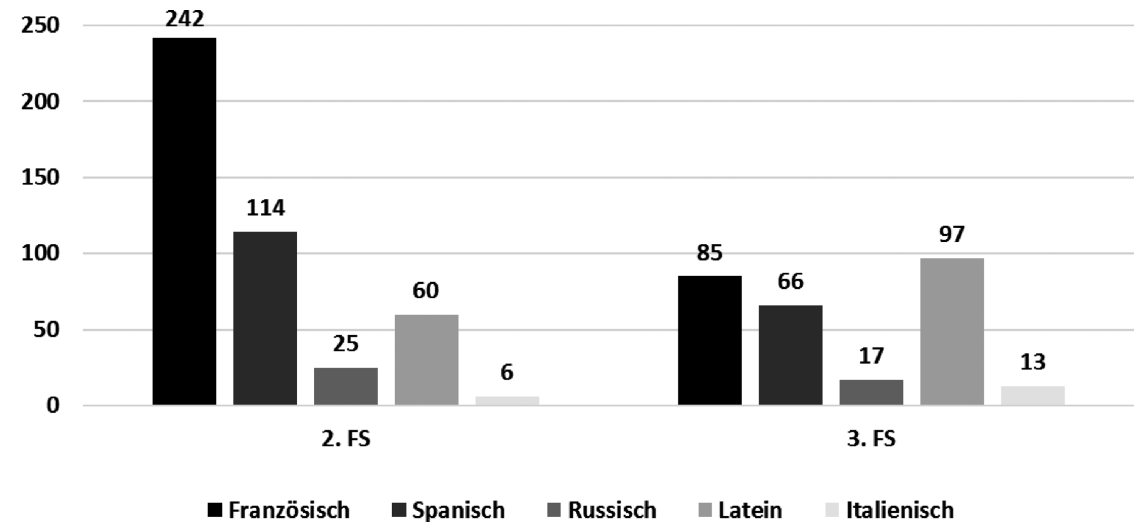

Abb. 5.2: Anzahl weiterführender Schulen in Berlin, die ausgewählte Sprachen als 2. oder 3. Fremdsprache anbieten. (Gezählt ist der frühestmögliche Zeitpunkt der Belegung einer Sprache, unabhängig davon, ob die Sprache auch später noch neu gewählt werden kann.) 
gesamten Sprachangebot hin. Es zeigt sich, dass Spanisch an dieser Stelle des Bildungsweges in Konkurrenz zu Französisch treten kann, das jedoch noch weitaus häufiger als zweite Fremdsprache angeboten wird. Gegenüber dem Lateinischen und auch dem Russischen hat Spanisch bereits jetzt einen Vorsprung. Latein wird öfter als dritte denn als zweite Fremdsprache angeboten, beim Spanischen ist es umgekehrt.

Insgesamt kann das Spanische inzwischen als etablierte Sprache im Schulsystem Berlins gelten. Ob es dauerhaft das Französische als zweitwichtigste Fremdsprache nach dem Englischen ablösen wird, ist fraglich. Die Entwicklungen etwa in Hamburg und Bremen lassen dies zumindest möglich erscheinen. Denkbar ist aber auch die Entwicklung hin zu einer weiter verbreiteten Wahl von insgesamt drei Fremdsprachen, so wie es die Anzahl der angebotenen Sprachen in sehr vielen weiterführenden Schulen ermöglicht. In diesem Fall dürfte die Kombination aus Englisch, Französisch und Spanisch sich zum Klassiker entwickeln, wobei die beiden romanischen Sprachen an zweiter und dritter Stelle in der Reihenfolge variabel sind und eine von beiden gelegentlich durch Latein ersetzt wird. Die Tatsache, dass das Englische als gesetzt gilt, darf allerdings nicht darüber hinwegtäuschen, dass es keineswegs automatisch die erste gelernte Fremdsprache sein muss. Im Unterschied zum Französischen kann Spanisch an weiterführenden Schulen in Berlin bisher aber in der Regel nicht als erste Fremdsprache gewählt werden. Es bleibt abzuwarten, ob sich dies angesichts der steigenden Nachfrage zukünftig ändert. Für manche Schulen könnte Spanisch als erste Fremdsprache ein attraktives Alleinstellungsmerkmal sein. 